\title{
A Contraction Mapping Method in Digital Image Processing
}

\author{
Ashutosh Mishra, Piyush Kumar Tripathi, A. K. Agrawal, Dev Raj Joshi
}

\begin{abstract}
The topological features of the objects or digital image pictures are characterized by digital topology. A digital image picture is a distinctive arrangement of numbers which are non negative. Decomposing an image picture into its constituent components and investigating its several characteristics with fundamental elements is generally coined as digital image processing. In investigating the fundamental constituents of images, the separation of connected segments are established to enquire the adjacency relationship. During this course of tracking, thinning and coding them, it is kept in mind that the specification of connectedness of the pictures remains unaltered.

The characteristics of the constituent subsets and relationships may be stipulated when the image is fragmented into its elementary constituents. Some features of the subsets of these constituent parts are based on their respective positions. Thus, the basic idea for image processing is the primary topological characteristics of digital images like adjacency, connectedness, etc.
\end{abstract}

The fixed point theorems associated to certain kinds of contraction mappings can be utilised in the field of engineering science and technology as computational technique to provide an exclusive programme to explore various problems. Parallel and distributed computation, modeling, simulation and digital image processing are few notables among these techniques.

In digital image processing digital contraction mapping is defined and then existence of the solution and its uniqueness is obtained using the fixed point theorem concerned, which is the mathematical basis of border following, thinning and contour filling of a digital image picture.

In digital image processing the applicability of fixed point theorem and contraction mappings as a computational technique has been grazed well.

To further broaden its pertinency in image processing our interest is to delve into some of contraction mappings as a significant mechanism.

Keywords: Digital Image, Digital Metric Space, Digital Contraction, Fixed Point Theorem.

\section{INTRODUCTION}

In functional analysis and general topology wide application of the contraction mapping is well understood. Also, it has been applied as a better problem solving tool in nonlinear analysis. A metric space with convergence of each Cauchy sequence lying in it confirms the existence of a fixed point with uniqueness when dealt with the contraction mapping. Metric space was brought into the notice by $\mathrm{M}$. Ferchet [1]. Further, Banach fixed point theorem introduced by Stephen Banach [3] paved a solid ground for the theory of

Revised Manuscript Received on December 5, 2019.

* Correspondence Author

Ashutosh Mishra, Amity University Uttar Pradesh (Department of Mathematics, Amity School of Applied Sciences)

Piyush Kumar Tripathi, Amity University Uttar Pradesh (Department of Mathematics, Amity School of Applied Sciences)

A. K. Agrawal, Amity University Uttar Pradesh (Department of Mathematics, Amity School of Applied Sciences)

Dev Raj Joshi, Amity University Uttar Pradesh (Department of Mathematics, Amity School of Applied Sciences) contraction mappings and their fixed points. Other researchers along with noted L.E.J. Brouwer [2], J. Schauder [4], etc., enhanced the notion as according. A. Tychonoff [5], S. Lefschetz [6], M. Edelstein [8], R. Kannan [9], etc., have further generalized the same notion. Distribution function has been furnished by K. Menger [7] in 1942 to provide an idea about the probabilistic metric space which is more helpful in dealing with the notion of metric space.

But, so far as digital topology is concerned, a digital metric space is taken in to account in place of general metric space. Likewise, a digital topology is used instead of using general topology in dealing with digital image processing.

\section{Digital Vs General Topology}

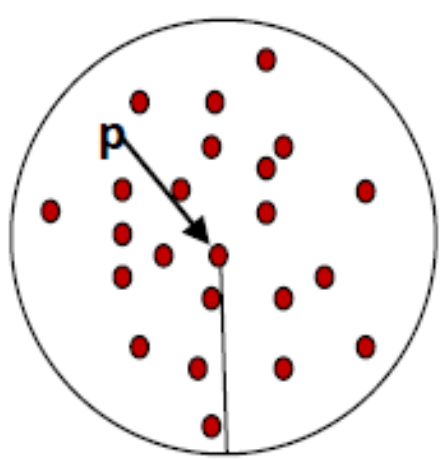

\section{General Topology: $N(p)=\infty$}

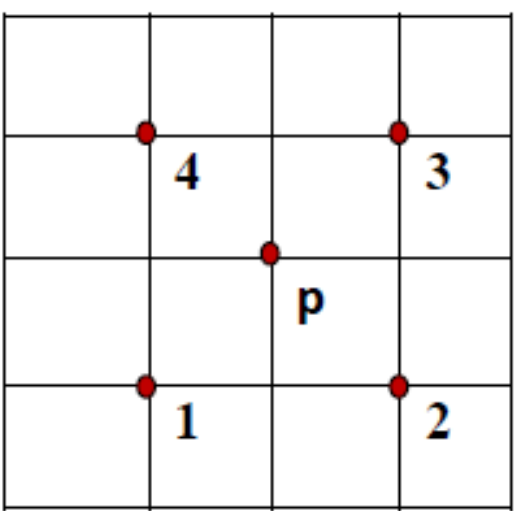

\section{Digital Topology: $N(p)=4$}

As stated above the general topology is not much appropriate while dealing with digital images since, it assumes the spaces which have uncounted number of points, even in the least neighborhood of the concerned space (as shown in above figures). 
So, many of the researchers have used digital topology which is the most appropriate topology in the course of image processing. It is nothing but the topological properties of two-dimensional (2D) and three-dimensional (3D) digital images. It furnishes a solid mathematical background for object counting, image thinning, contour filling and various other image processing techniques.

Digital topology has been considered as an important tool of image processing by A. Rosenfield [10] [11]. Then, the plan of digital fundamental group for a three-dimensional image thinning was suggested by Kong [12]. After studying a number of digital continuous mappings, the idea of digital topology has been forwarded by Boxer [14] [16] [19]. It was Rosenfield [11] who first proposed the concept of digital continuous function and characteristics of fixed point in digital images.

Ege and Karaca [25] [26] advanced the applicability of Lefchetz fixed point theorem for the processing of digital images and defined the concept of digital metric space to prove the Banach contraction theorem for digital images [27]. Further improvement of the Banach theorem for digital metric was fostered by Han [28].

Our wish in this is to further strengthen the notion of digital topology by procuring some contractions in processing the digital images.

\section{Preliminaries}

Before dealing with the main purpose, it is needful to furnish some basics.

Suppose any subset $L$ of the set $Z^{n}$ of lattice pointsin $n$-dimensional Euclidean space, where $n$ is an integer such that $n \geq 0$ and the adjacency relation for the elements of $L$ is a.Then, $(L, a)$ is known as a digital image [10] [11].

Definition 1: For some positive integers $a, n ; 1 \leq a \leq n$ and two fixed points $p, q$ s. t.

$p=\left(p_{1}, p_{2}, \ldots \ldots \ldots p_{\mathrm{n}}\right), q=\left(q_{1}, q_{2}, \ldots \ldots \ldots q_{\mathrm{n}}\right) \in Z^{n}$, then $p, q$ are $a$-adjacent [16] if for at most $a$, we get

$\left|p_{i}-q_{i}\right|=1$, for all $i$ and $\left|p_{j}-q_{j}\right| \neq 1, p_{j}=q_{j}$ for all other indices $j$.

So, we get

For $p \in Z^{n}$ number of points $q \in Z^{n}$ adjacent to $p$ can be depicted by $k(a, n)$ where $k(a, n)$ is independent to $p$. Or, we use $k=k(p, n)$ in short.

Now, follow this as:

(i) When, $p \in Z$, i.e., $n=1$, so $a$ will have the value $a=1$. Therefore,

$$
k(a, n)=k(1,1)=2
$$

In this case the two points are $p-1$ and $p+1,1$-adjacent to $p \in Z$ which is as shown in figure below.

\section{1-adjacency}

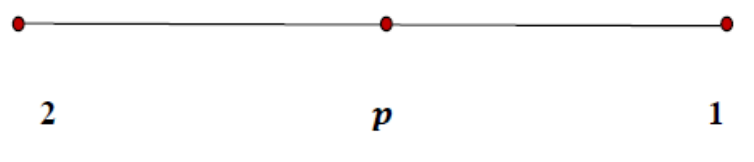

(ii)When, $p \in Z^{2}, n=2$, so $a$ will have the values $a=1,2$. If $a=2$. Then, 2 -adjacent to $p=\left(p_{1}, p_{2}\right)$ will be

$$
\left(p_{1} \pm 1, p_{2}\right),\left(p_{1}, p_{2} \pm 1\right),\left(p_{1} \pm 1, p_{2} \pm 1\right) \text {. }
$$

So, there are 8 points 2 -adjacent to $p$ such that $k=k(2,2)=$ 8.

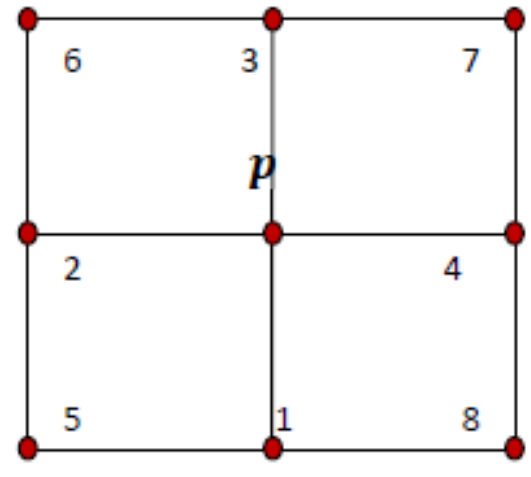

\section{2-adjacency}

If $a=1$. Then, 1 -adjacent to $p=\left(p_{1}, p_{2}\right)$ will be $\left(p_{1} \pm\right.$ $1, p 2, p 1, p 2 \pm 1$.

Therefore, there are 4 points 2 -adjacent to $p$ such that $k=k(1,2)=4$.

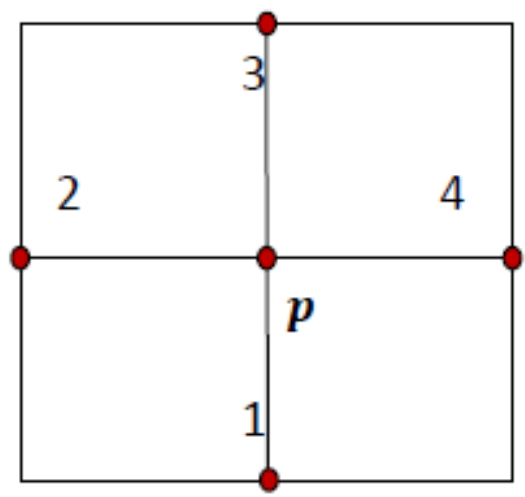

\section{1-adjacency}

(iii)If, $p \in Z^{3}, n=3$, so $a$ will have the values $a=1,2,3$. When, $p=3$. Then, 3 -adjacent to $p=\left(p_{1}, p_{2}, p_{3}\right)$ are given by

$$
\begin{gathered}
\left(p_{1} \pm 1, p_{2}, p_{3}\right),\left(p_{1}, p_{2} \pm 1, p_{3}\right),\left(p_{1}, p_{2}, p_{3} \pm 1\right), \\
\left(p_{1} \pm 1, p_{2} \pm 1, p_{3}\right),\left(p_{1} \pm 1, p_{2}, p_{3} \pm 1\right),\left(p_{1}, p_{2} \pm 1, p_{3}\right. \\
\pm 1), \\
\left(p_{1} \pm 1, p_{2} \pm 1, p_{3} \pm 1\right)
\end{gathered}
$$

Therefore, there are 26 points 3 -adjacent to $p$ such that $k=k(3,3)=26$.

If $a=2$. Then, 2 -adjacent to $p=\left(p_{1}, p_{2}, p_{3}\right)$ are given by

$$
\begin{gathered}
\left(p_{1} \pm 1, p_{2}, p_{3}\right),\left(p_{1}, p_{2} \pm 1, p_{3}\right),\left(p_{1}, p_{2}, p_{3} \pm 1\right), \\
\left(p_{1} \pm 1, p_{2} \pm 1, p_{3}\right),\left(p_{1} \pm 1, p_{2}, p_{3} \pm 1\right),\left(p_{1}, p_{2} \pm 1, p_{3}\right.
\end{gathered}
$$$$
\pm 1)
$$

Therefore, there are 18 points 2 -adjacent to $p$ such that $k=k(2,3)=18$.

If $a=1$. Then, 1 -adjacent to $p=\left(p_{1}, p_{2}, p_{3}\right)$ are given by

$$
\left(p_{1} \pm 1, p_{2}, p_{3}\right),\left(p_{1}, p_{2} \pm 1, p_{3}\right),\left(p_{1}, p_{2}, p_{3} \pm 1\right)
$$

Therefore, there are 6 points 1 -adjacent to $p$ such that $k=k(1,3)=6$.

Hence, for $\mathrm{n}-\mathrm{D}$ digital image it can be generalized as shown by the following equation [18]. 


$$
k(a, n)=\sum_{i=n-a}^{n-1} 2^{n-i}\left(\begin{array}{l}
n \\
i
\end{array}\right), 1 \leq a \leq n
$$

Where,

$$
\left(\begin{array}{l}
n \\
i
\end{array}\right)=\frac{n !}{i !(n-i) !}
$$

Consider a non empty set $L$, subset of $Z^{n}$, s.t. $1 \leq a \leq n$ and $k=k(a, n)$. Then, $k(a, n)$ is said to be a digital image with $a$-adjacency [11]. And, the pair $(L, a)$ is the n-D digital image [10] [11].

Definition 2: [14] [15] A digital interval denoted by $[l, m]_{z}$ for $l, m \in Z$ and $l<m$ is defined as

$$
[l, m]_{z}=\{z \in Z: l \leq z \leq m\} \text {. }
$$

Definition 3: [15] Any point of $Z^{n}$ which is $a$-adjacent to $p$ is known as $a$-neighbour of $p \in Z^{n}$ if for $a \in\{2,4,8,6,18,26\}$ and $n \in\{1,2,3\}$, we have

$$
M_{a}(\mathrm{p})=\{\mathrm{q}: \mathrm{q} \text { is } a \text {-adjacent to } p\}
$$

$M_{a}(\mathrm{p})$ is an $a$-neighbourhood to $a$-neighbour of $p$. Here, $p$ and $q$ are $a$-neighbours if $\mathrm{q}$ is $a$-adjacent to $p$.

Definition 4: [15] An $a$-connected digital image $L \in Z^{n}$ is described for every points $l, m \in L$ if $\exists$ a set $\left\{l_{0}, l_{1}, l_{2}, \ldots \ldots \ldots, l_{r}\right\}$, such that $l=l_{0}$ and $m=m_{r} \& l_{i}$ and $l_{i+1}$ are $a$-neighbours $\forall i=0,1,2, \ldots \ldots \ldots, r-1$.

Definition 5: Consider the digital images $\left(\mathrm{L}, a_{0}\right)$ and $\left(\mathrm{N}, a_{1}\right)$ of $Z^{n_{0}}$ and $Z^{n_{1}}$, respectively and $B: L \rightarrow N$ is a Function. Then

(i) $B$ is $\left(a_{0}, a_{1}\right)$-continuous [16] if $a_{0}$-connected subsets $F$ of $L$ are corresponded to $a_{1}$-connected to $N$.

(ii) $B$ is $\left(a_{0}, a_{1}\right)$-continuous if and only if images of $a_{0}$-adjacent to $L$ are $a_{1}$-adjacent in $N$ else, images of $a_{0}$-adjacent of $L$ are coinciding. Meaning there by, $l_{0}, l_{1}$ are $a_{0}$-adjacent to $L$ then, $B\left(l_{0}\right)$ and $B\left(l_{1}\right)$ are $a_{1}$-adjacent in $N$ or, $B\left(l_{0}\right)=B\left(l_{1}\right)$.

(iii)Isomorphism: [20] $B$ is called $\left(a_{0}, a_{1}\right)$-isomorphism if :

(a) $B$ is $\left(a_{0}, a_{1}\right)$-continuous

(b) $B$ is one-one and onto function and

(c) $B^{-1}$ is $\left(a_{0}, a_{1}\right)$-continuous.

Then, we obtain

$$
L \cong_{\left(a_{0}, a_{1}\right)} \mathrm{N} \text {. }
$$

Definition 6: [16] A Digital $a$-path from $l \rightarrow m$ in $L$ is defined as a $(2, \mathrm{a})$-continuous function $\mathrm{B}:[0, r]_{z} \rightarrow L \mathrm{~s}$. $\mathrm{t}$. $B(0)=l$ and $B(\mathrm{r})=m$. The set $L$ is known as $a$-path connected in the set $(L, a)$ if there is ana-path for any two points.

A simple closed $a$-curve of $r \geq 4$ points [22] in $(L, a)$ is a specified by a sequence $\{B(0), B(1), B(2), \ldots \ldots \ldots, B(\mathrm{r}-$ $1\}$ of $a$-path images $\mathrm{B}:[0, r] z \rightarrow L$ such that $B$ and $B]$ are $a$-adjacent if and only if $j=i \pm \bmod r$.

\section{Fixed point theorem for digital images:}

Suppose $B:(L, a) \rightarrow(L, a)$ is a $(\mathrm{a}, \mathrm{a})$-continuous self map. Any digital image $(L, a)$ have a fixed point [26] if $\exists \alpha \in$ Lsuch that for every $(\mathrm{a}, \mathrm{a})$-continuous mapping $B: L \rightarrow L$

$$
B(\alpha)=\alpha
$$

The characteristics shown above are protected in any digital isomorphism for fixed point.

Now, let $(L, b, a)$ is a digital metric spacewith $a$-adjacency and $b$ an Euclidean metric on $Z^{n}$.

Definition 7: [25] [26] [27] Consider $\left\langle\alpha_{n}\right\rangle$ is a sequenceon a digital metric $(L, b, a)$. The sequence $\left\langle\alpha_{n}\right\rangle$ will be a Cauchy sequence, if for each $\varepsilon>0 \exists c \in N$, we have

$$
b\left(\alpha_{r}, \alpha_{n}\right)<\varepsilon, \forall r, n>c .
$$

Theorem 1: [28] If $<\alpha_{n}>$ is a Cauchy sequenceon a digital $\operatorname{metric}(L, b, a)$ then, we have

$$
\alpha_{r}=\alpha_{n}, \forall r, n>c \in N
$$

Definition 8: [28] A Cauchy sequence $\left\langle\alpha_{n}>\right.$ on a digital digital metric $(L, b, a)$ is convergent on $\alpha_{0} \in \mathrm{L}$, if for each $\varepsilon>0 \exists c \in N$, such that

$$
b\left(\alpha_{n}, \alpha_{0}\right)<\varepsilon \forall n>c
$$

Definition 9: [28] A digital metric space $(L, b, a)$ is complete if every Cauchy sequence $\left\langle\alpha_{n}>\right.$ on the metric space $(L, b, a)$ is convergent to any point $\alpha_{0} \in \mathrm{L}$.

Theorem 2: [28] Let $(L, b, a)$ is anydigital metric space then the digital metric space will be complete.

Definition 10: [28] Let $B:(L, b, a) \rightarrow(L, b, a)$ be a self map on a given digital metric $(L, b, a)$. Then, for any $\beta \in[0,1)$ it will be a digital contraction if,

$$
b(B l, B m) \leq \beta b(l, m), \forall l, m \in L .
$$

Where, $\beta$ is a digital contraction constant.

Statement 1: [28] Each digital contraction mapping $B:(L, b, a) \rightarrow(L, b, a)$ on a digital metric $(L, b, a)$ is always digitally continuous.

Now, before going through the main result we recall the digital contraction for self mappings.

Theorem 3: [27] Banach Contraction Principle

Suppose $(L, b, a)$ is a digital metric space on Euclidean metric $Z^{n}$ and $B: K \rightarrow K$ be any digital contraction. Then, a unique fixed point $x_{0} \in L$ exists, which gives $B\left(x_{0}\right)=x_{0}$.

\section{MAIN RESULTS AND DISCUSSION}

The key motive of this draft is to explore the pertinency of contraction mapping principal in digital topology especially the inference of this paper is to broaden the applicability of fixed point theorems in digital image processing.

Now, we use two mappings to show the main result as given here under with the following theorem:

Theorem 4: Let $(L, b, a)$ be a digital metric space with coefficient $k \geq 1$ and $f, g: D \rightarrow L$ the mapping such that

\footnotetext{
$b\left(f x_{x}, f y\right) \geq h \cdot \min \left\{b(g x, g y), b(f y, g y), \frac{1}{2 k}[b(g x, f y)+b(g y, f x)]\right\}$ where for all $x, y \in X k h<1$

ii. Either $f(D)$ or $g(D)$ is complete then $f$ and $g$ have a coincidence point and hence fixed pint.
}

Proof: Let $x_{0}, x_{1}$ be points of $Y$ such that $f x_{0}=g x_{1}$. Since, $f(D) \subseteq g(D)$ and $f x_{0}=g x_{1}$, hence we can construct a sequence $\left\{x_{n}\right\}$ such that $f x_{n}=g x_{n+1}$ in (i) we have

$$
\begin{aligned}
& b\left(f_{x}, f x_{n+1}\right)=b\left(z_{n}, z_{n+1}\right) \\
& \geq h \cdot \min \{b(g x, g y), b(g x, f x)\}, b \frac{1}{2 k}[b(g x, f y) \\
& +b(g x, f x)]
\end{aligned}
$$

$\geq$

h. $\min \left\{b\left(g_{n}, g x_{n+1}\right), b\left(g x_{n}, f x_{n}\right), b\left(g x_{n+1}, f x_{n}\right)\right\}$,

$\frac{1}{2 k}\left[b\left(g x_{n}, f x_{n+1}\right)+b\left(g x_{n+1}, f x_{n}\right)\right]$

Let $z_{n}=f x_{n}=g x_{n+1}=g x_{n}=f x_{n-1}=z_{n-1}$. Then $b\left(z, z_{n+1}\right) \geq h \cdot \min g\left(z_{n-1}, z_{n}\right), b\left(z_{n}, z_{n-1}\right.$

$$
\begin{aligned}
& ), \mathrm{b}\left(z_{n-1}, z_{n}\right), \mathrm{b}\left(z_{n}, z_{n+1}\right)\right\}, \frac{1}{2 k}\left[b\left(z_{n-1}, z_{n}\right), b\left(z_{n-1}, z_{n}\right)\right] \\
& \geq h \cdot \min \left\{b\left(z_{n-1}, z_{n}\right), b\left(z_{n-1}, z_{n}\right), b\left(z_{n}, z_{n+1}\right), b\left(z_{n-1}, z_{n}\right)\right\}
\end{aligned}
$$

When $k=1$, we have $b\left(z_{n-1}, z_{n}\right)<b\left(z_{n}, z_{n+1}\right)$, $b\left(z_{n+1}, z_{n}\right) \leq h \cdot b\left(z_{n-1}, z_{n}\right)$, 
By using triangle inequality of digital metric space

So, we get $\left\{z_{n}\right\}=f x_{n}$ is a Cauchy sequence. Suppose $g(D)$ is complete.

Then $\exists p \in g(D)$

Such that, $z_{n} \rightarrow p$ and $\exists z \in D$

Such that, $g_{z}=p$.

Putting $x=x_{n}$ and $y=z$,we have,

$b\left(f x_{n}, f z\right)$

$\geq h \min \left\{b\left(g x_{n}, g_{z}\right), b\left(g x_{n}, f x_{n}\right), b(g z, f z) \frac{1}{2 k}\left[b\left(g x_{n}, f z\right)\right.\right.$

$\left.\left.+b\left(g z, f x_{n}\right)\right]\right\}$

Taking limit $n \rightarrow \infty$, we get,

$$
\begin{gathered}
b(p, f z) \geq h \min \left\{\mathrm{b}(p, f z), \mathrm{b}(\mathrm{p}, \mathrm{p}), \mathrm{b}(g z, f z), \frac{1}{2 k}[b(p, f z)\right. \\
+b(z, p)] \\
\begin{aligned}
b\left(p, f_{z}\right) \geq h \min \left\{b(p, p), b(p, p), b(f z, p), \frac{1}{2 k}[b(p, f z)\right. \\
+
\end{aligned} \\
b(g z, p)] \\
b\left(p, f_{z}\right) \geq h \min \left\{b(f z, p), \frac{1}{2 k} b(p, f z)\right\}
\end{gathered}
$$

And $b(p, f z) \geq h \min b(p, f z)=0$

By triangle inequality this is possible only if $b(p, f z)=0$, so $p=f(z)=p(z)$. Hence, $p$ is a coincidence point of $f$ and $g$. Again, if we consider $Y=X$ then we can easily prove $p$ is a common fixed point of $f$ and $g$.

\section{CONCLUSION}

Though, fixed point theorems have range of uses but, in the field of computer science and technology it has been probed an important tool. In image processing, fixed point theorems may easily be used in reducing the size and enhancing the quality of the image by using contraction principle. It is seen that the quality of the compressed image after processing is not obtained so good every time even, it could not carry the same information as the image source. Sometimes, the memory of the obtained data is too very large which is an, another type of headache while storing them. Nonetheless, digital image compression is the need of the moment.

Thus, using fixed point theorem, as shown in the main result, we may reinforce the idea of digital topology especially, in image processing to compress the image with less redundancy.

\section{ACKNOWLEDGEMENT}

The valuable suggestions for improvement of the notion stated in the paper concerned would be solicited and thanked with core of the heart. Above all, authors are keen to render their gratitude towards The Almighty for providing the the chance.

\section{REFERENCES}

1. Frechet, M. (1906), "Sur quelques points du calculfonctionnel", Rendiconti del Circolomatematico di Palermo,Vol. 22, No.1,pp.1-72.

2. Brouwer, L. E. J. (1912), "Uber abbildung von mannigfaltigkiten, mathematische annalen", vol. 71, pp. 97-115.

3. Banach, S. (1922), Sur les opérations dans les ensembles abstraits et leurs applications aux équations intégrals. Fundam. Math. 3, 133-181.

4. Schauder, J. (1930), Der fixpunktraz functional saumen, Studia Math. $2,171-180$

5. Tychonoff, A. (1935), "Ein fixpunktsatz", Math. Ann., vol. 111, pp. 767-776.
6. Lefschetz, S. (1937), "On the fixed point formula" Ann. of Math. vol. 38 , no. 4, pp. 819-822.

7. Menger, K. (1942), Statistical metric, Proc. Nat. Acad. (USA) 28, 535

8. Edelstein, M. (1960), "An extension of Banach's contraction principle”, Proc. Amer. Math. Soc., vol. 12, pp.7-10.

9. Kannan, R. (1968) "Some results on fixed points", Bull. Calcutta Math. Soc., vol. 60, pp.71-76.

10. Rosenfeld, A. (1979), Digital Topology, Amer. Math. Monthly, vol.86, No.8, pp.621-630.

11. Rosenfeld, A. (1986), Continuous functions on digital pictures, Pattern Recognition Letters 4, pp. 177-184

12. Kong, T. Y. (1989), A Digital Fundamental Group, Computers and Graphics, 13, 159-166.

13. Hicks, T. L. (1992), Fixed point theorems for d - complete topological spaces 1, Internat. J. Math. and Mat. Sci., vol.15, No.3, pp.435-440.

14. Boxer, L. (1994), Digitally Continuous Functions, Pattern Recognition Letters, 15, 833-839.

15. Kong, T. Y., Rosenfeld, A. (1996), Topology Algorithms for the digital Image Processing, Elsevier Sci., Amsterdam.

16. Boxer, L. (1999), A Classical Constructions for The Digital Fundamental Group, J. Math. Imaging Vis., 10, 51-62.

17. Sastry, K. P. R. and Srinivasa Rao, C. H. (2000), A unique fixed point theorem for a self map on $\mathrm{F}$ - complete topological spaces, Bull. Cal. Math. Soc., 92(1), 71-74.

18. Han, S. E. (2005), Non-Product property of the digital fundamental group, Inform. Sci., 171, pp. 73-91.

19. Boxer, L. (2005), Properties of Digital Homotopy, J. Math. Imaging Vis., 22(2005), 19-26.

20. Boxer, L. (2006), Digital products, wedges and covering spaces, Journal of Mathematical Imaging and Vision 25, 159-171.

21. Boxer, L. (2006), Homotopy properties of sphere-like digital images. J. Math. Imaging Vis. 24, 167-175.

22. Boxer, L. (2010), Continuous maps on digital simple closed curves, Appl. Math. 1, 377-386.

23. Peeler, Luke (2011), Metric Spaces and the contraction mapping principle, Math. Unchicago, edu.

24. Choudhury, B. S., Kumar, S., Asha, K. Das (2012), Some fixed point theorems in G-metric spaces, Math. Sci. Lett. 1(1), 25-31

25. Ege, O., Karaca, I. (2013), Lefschetz Fixed Point Theorem for DigitalImages, Fixed Point Theory.Appl., 13 pages.

26. Ege, O., Karaca, I. (2014), Applications of The Lefschetz Number to Digital Images, Bull. Belg. Math. Soc. Simon Stevin, 21, 823-839.

27. Ege, O., Karaca, I. (2015), Banach Fixed Point Theorem for Digital Images, J. Nonlinear Sci. Appl. 8, 237-245.

28. Han, S. E. (2016), Banach fixed point theorem from the viewpoint of digital topology, J. Nonlinear sci. Appl., 9, 895-905. 